\title{
COPD sleep phenotypes: Genesis of respiratory failure in COPD
}

\author{
Sameer Vaidya, Dipti Gothi, Mahismita Patro \\ Department of Pulmonary Medicine, ESI Hospital Basai Darpur, New Delhi, India
}

\begin{abstract}
The chronic obstructive pulmonary disease (COPD) patients could have respiratory failure during sleep without daytime overt arterial blood gas (ABG) abnormality. We undertook a study first of its kind to attempt in distinguishing the underlying pathophysiological mechanisms. It was a prospective observational study in stable COPD patients. The inclusion criterion was presence of day time $\mathrm{PaO}_{2}>60 \mathrm{mmHg}$ and $\mathrm{PaCO}_{2}<45 \mathrm{mmHg}$. Twenty five out of 110 patients were excluded because of the ABG abnormality. The remaining 85 patients were subjected to overnight pulse oximetry and end-tidal (ET)- $\mathrm{CO}_{2}$ monitoring. The nocturnal oxygen desaturation was defined as per Fletcher's criteria. The nocturnal hypoventilation was defined as per American academy of sleep medicine (AASM) guidelines. Patients having saw-tooth pattern
\end{abstract}

Correspondence: Dr Dipti Gothi, Professor, Department of Pulmonary Medicine, ESI Hospital Basai Darpur, New Delhi, India.

Tel. +91.9971550550.

E-mail: diptigothi@gmail.com

Key words: COPD phenotypes; OSA; nocturnal hypoventilation; noc-turnal oxygen desaturation.

Ethics approval and consent to participate: The study was carried out after obtaining ethical clearance in adherence to the guidelines of the declaration of Helsinki from the institutional ethical committee (IEC PGIMSR Basai Darapur, New Delhi, No. DM (A) H-19/14/17/IEC/2012-PGIMSR dated 27/11/2017). Written and informed consent was obtained from all the participants.

Conflict of interest: The authors declare that they have no competing interests, and all authors confirm accuracy.

Acknowledgements: We sincerely acknowledge the hard work of our sleep technicians, without whom this study would not have been possible.

Contributions: DG, guarantor. All the authors made a substantive intellectual contribution, read and approved the final version of the manuscript and agreed to be accountable for all aspects of the work

Received for publication: 22 January 2021

Accepted for publication: 10 August 2021.

${ }^{\circ}$ Copyright: the Author(s), 2021

Licensee PAGEPress, Italy

Monaldi Archives for Chest Disease 2022; 92:1776

doi: 10.4081/monaldi.2021.1776

This article is distributed under the terms of the Creative Commons Attribution Noncommercial License (by-nc 4.0) which permits any noncommercial use, distribution, and reproduction in any medium, provided the original author(s) and source are credited. on pulse oximetry and/or snoring were subjected to polysomnography. Thirty-eight patients out of $85(44.8 \%)$ had nocturnal gas exchange abnormality in absence of daytime respiratory failure and were identified into 3 different phenotypes: obstructive sleep apnea (OSA), nocturnal hypoventilation and nocturnal oxygen desaturation. The isolated abnormality was seen in 24 patients: 10 patients had OSA, 9 had nocturnal hypoventilation and 5 had nocturnal oxygen desaturation. Overlap of two or more phenotypes was seen in 14 patients. As compared to the nocturnal hypoventilation and desaturation phenotypes, the OSA phenotype had a significantly higher BMI and $\mathrm{FEV}_{1}$. The nocturnal hypoventilation and the desaturation phenotypes did not have significant difference in $\mathrm{FEV}_{1}$ and $\mathrm{BMI}$, but the daytime $\mathrm{SpO}_{2}$ and $\mathrm{PaO}_{2}$ differed significantly. Such parameters could help in identifying the three distinct COPD-sleep phenotypes (OSA, nocturnal hypoventilation and nocturnal oxygen desaturation). A phenotype based nocturnal management may help in delaying the process of overt respiratory failure in COPD.

\section{Introduction}

Chronic obstructive pulmonary disease (COPD) is a disease with a high global morbidity and mortality burden. The high morbidity and mortality are primarily related to chronic respiratory failure. The COPD patients have impaired nocturnal respiratory disturbance prior to developing overt chronic respiratory failure $[1,2]$. There have been no studies so far which have evaluated nocturnal gas exchange abnormality in absence of overt respiratory failure in COPD with an attempt to distinguish the underlying pathophysiological mechanisms. The COPD patients may develop nocturnal hypoxemia and/or nocturnal hypoventilation. The hypoxia is caused by the ventilation perfusion mismatch and hyperinflation of the lung [3]. The hypoventilation is caused by pump failure because of the impaired central drive and the blunted chemoreceptor response [4]. Furthermore, COPD patients could have co-occurrence of obstructive sleep apnea (OSA) that can also cause nocturnal gas exchange abnormality [5]. So far, no study has segregated these respiratory abnormalities and differentiated the clinical characteristics. We undertook this study to classify COPD patients into distinct sleep phenotypes in order to identify the clinical characteristics for its early recognition and appropriate management.

\section{Aims and objectives}

To find out the prevalence of respiratory disturbance at night in absence of daytime respiratory failure and to identify and characterize "COPD sleep phenotypes". 


\section{Materials and Methods}

This was a prospective observational study, carried out in a tertiary care center. The study was carried out after obtaining ethical clearance in adherence to the guidelines of the declaration of Helsinki from the Institutional Ethical Committee (IEC PGIMSR Basai Darapur, New Delhi, No. DM (A) H-19/14/17/IEC/2012PGIMSR dated 27/11/2017) and written informed consent from the patients. 110 stable COPD patients coming to the outpatient department from December 2017 to July 2018 were enrolled in the study. As per the department protocol spirometry was performed for all the subjects with Medisoft/Morgan Scientific Spiro Airin ${ }^{\circledR}$ and was interpreted as per the American Thoracic Society (ATS) guidelines [6]. The forced vital capacity (FVC), forced expiratory volume at 1 second $\left(\mathrm{FEV}_{1}\right), \mathrm{FEV}_{1} / \mathrm{FVC}$ ratio were recorded. The patients were diagnosed COPD according to The Global Initiative for Chronic Obstructive Lung Disease (GOLD) definition [7]. The daytime arterial blood gas $(\mathrm{ABG})$ analysis was performed at the outset. The patients who had abnormal day time $\mathrm{ABG}$ i.e., $\mathrm{PaO}_{2}<60 \mathrm{mmHg}$ and $\mathrm{PaCO}_{2}>45 \mathrm{mmHg}$ were excluded from study. Patients with cardiac failure or ejection fraction $<50 \%$ were also excluded.

The demographic features like age, sex and body mass index (BMI) of remaining 85 patients were noted down. Detailed clinical history and examination findings were noted. All the patients were thoroughly evaluated with respect to their sleep. This included the total duration of sleep, quality of sleep and day time symptoms. Snoring history was elicited from the patient as well as close relatives.

All the patients were subjected to unsupervised overnight pulse oximetry and overnight end tidal $\mathrm{CO}_{2}\left(\mathrm{ET}-\mathrm{CO}_{2}\right)$ monitoring in the sleep laboratory. The sleep laboratory was maintained as per the American academy of sleep medicine (AASM) criteria [8]. The pulse oximetry was done using a finger probe. The ET$\mathrm{CO}_{2}$ was measured using a nasal cannula. The finger probe and nasal cannula were attached to the polysomnography machine. All the patients were subjected to a second daytime ABG analysis at the start of the study. The values of $\mathrm{ABG}$ were correlated with the $\mathrm{SpO}_{2}$ and the ET-CO .

Those patients who gave history of snoring and/or saw tooth pattern on overnight pulse oximetry [9] were subjected to level 1 polysomnography (PSG) as per the AASM guidelines [8]. Philips Respironics Alice 5 Diagnostic Sleep System ${ }^{\circledR}$ was used. The scoring of sleep stage and the respiratory events were done as per the AASM guidelines [8]. 20 patients were subjected to overnight polysomnography based on history of snoring and saw tooth pattern on nocturnal pulse oximetry.

The data was tabulated on MS-Excel ${ }^{\circledR}$ (2010). Statistical analysis for mean, standard deviation, sensitivity and specificity was done using open EPI software ${ }^{\circledR}$ (OpenEpi: Open Source Epidemiologic Statistics for Public Health, Version 3.01). The pvalue was calculated using $t$-test for the continuous variables and Kruskal Wallis test for non-parametric variables.

\section{Definitions}

OSA: Five or more scoreable respiratory events (apnea or hypopneas) per hour of sleep along with daytime or nocturnal symptoms or rather fifteen or more scoreable respiratory events (apneas or hypopneas) per hour of sleep [9].

Nocturnal hypoventilation: An increase in the ET- $\mathrm{CO}_{2}$ to $>55$ $\mathrm{mm} \mathrm{Hg}$ for $\geq 10 \mathrm{~min}$, or an increase in the ET- $\mathrm{CO}_{2}$ by $\geq 10 \mathrm{~mm} \mathrm{Hg}$ above the awake supine value to a value over $50 \mathrm{~mm} \mathrm{Hg}$ for $\geq 10$ $\min [10]$.

Nocturnal oxygen desaturation: A drop in $\mathrm{SpO}_{2}$ to $<90 \%$ for $>30 \%$ of total sleep time without nocturnal $\mathrm{ET}-\mathrm{CO}_{2}$ increase [11].

\section{Results}

Among the 110 COPD, 25 patients were found to have deranged day-time blood gases i.e., $\mathrm{PaO}_{2}<60 \mathrm{mmHg}$ and $\mathrm{PaCO}_{2}$ $>45 \mathrm{mmHg}$. The remaining 85 patients were included in the study. The average age was $59.6 \pm 7.69$ years. $82 / 85$ patients had COPD secondary to cigarette/bidi smoking and 3 had exposure to environmental smoke. 38/85 (44.8\%) patients were found to have nocturnal gas exchange abnormality. These patients could be identified into 3 different phenotypes. These phenotypes were seen either in isolation or as overlap of two or more. The isolated abnormality was seen in 24 patients: 10 patients had OSA, 9 had nocturnal hypoventilation and 5 had nocturnal oxygen desaturation. An overlap of two or more phenotype was seen in 14 patients (Figure 1). 7 patients had nocturnal oxygen desaturation and nocturnal hypoventilation. 2 patients had OSA and nocturnal hypoventilation. Another 2 had OSA and nocturnal oxygen desaturation. Three patients had all 3 disorders simultaneously, i.e., OSA with nocturnal oxygen desaturation and nocturnal hypoventilation. The mean nocturnal $\mathrm{SpO}_{2}$, mean nocturnal $\mathrm{ET}-\mathrm{CO}_{2}$, mean night-time T90 ( $\%$ time in bed with $\mathrm{SpO}_{2}<90 \%$ ), mean daytime $\mathrm{SpO}_{2}$, mean day-time $\mathrm{PaO}_{2}$, mean $\mathrm{FEV}_{1}$ (in $\mathrm{ml}$ and \% predicted), and mean BMI values in the isolated phenotypes are given in Table 1. Table 2 shows the statistical significance of the differences in above parameters in the three COPD-sleep phenotypes and their interpretation.

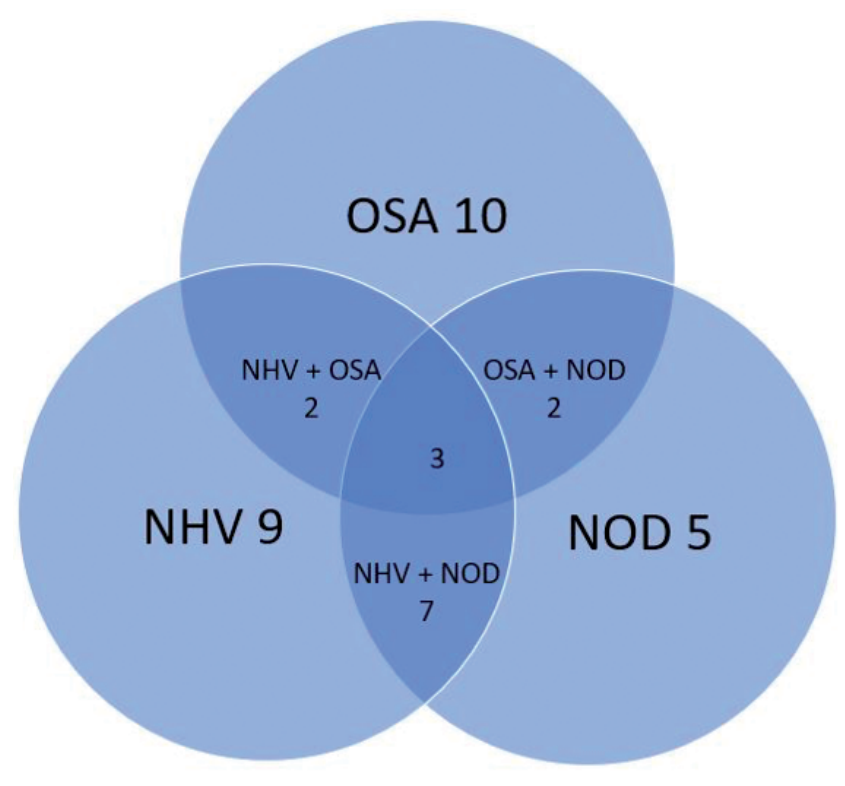

Figure 1. Venn diagram showing COPD sleep phenotypes. OSA, obstructive sleep apnea; NOD, nocturnal oxygen desaturation; NHV, nocturnal hypoventilation. 
The nocturnal oxygen desaturation phenotype was characterized by lower nocturnal $\mathrm{SpO}_{2}$ and higher night time T90. The nocturnal hypoventilation phenotype had higher nocturnal ET- $\mathrm{CO}_{2}$. The nocturnal oxygen desaturation and OSA phenotypes had significantly lower daytime $\mathrm{SpO}_{2}$ and $\mathrm{PaO}_{2}$ compared to nocturnal hypoventilation phenotype. The lung functions and BMI were significantly higher in OSA phenotype compared to other phenotypes. The distinguishing characteristics of the three COPD-sleep phenotypes are shown in Figure 2.

\section{Discussion}

McNicholas et al. stated in 2013 that sleep is a forgotten dimension in COPD [1], it still remains so. The COPD patients are not routinely evaluated in terms of nocturnal gas exchange abnormality. We found that $44.8 \%$ patients had nocturnal gas exchange abnormality when there was no daytime chronic respiratory failure. The nocturnal hypoxemia was shown in a study by Fletcher et al. [12] where

Table 1. Mean nocturnal $\mathrm{SpO}_{2}$, mean nocturnal ET- $\mathrm{CO}_{2}$, mean night-time T90 (\% TIB), mean day-time $\mathrm{SpO}_{2}$, mean day-time $\mathrm{PaO}$, mean $\mathrm{FEV}_{1}$ (in $\mathrm{ml}$ and \% predicted), and mean BMI values in the various COPD-sleep phenotypes.

\begin{tabular}{|c|c|c|c|}
\hline COPD-sleep phenotype & OSA (10) & Nocturnal hypoventilation (9) & Nocturnal oxygen desaturation (5) \\
\hline Mean nocturnal $\mathrm{SpO}_{2}$ & $93.2 \pm 2.01$ & $95.12 \pm 2.47$ & $90.4 \pm 2.96$ \\
\hline Mean nocturnal ET-CO & $42.3 \pm 1.82$ & $45.25 \pm 1.28$ & $40.6 \pm 1.51$ \\
\hline Mean night-time T90 (\% TIB) & $14.4 \pm 3.77$ & $3.17 \pm 4.82$ & $42.33 \pm 16.93$ \\
\hline Mean daytime $\mathrm{SpO}_{2}(\%)$ & $93.9 \pm 2.5$ & $97.22 \pm 0.66$ & $95.4 \pm 1.14$ \\
\hline Mean daytime $\mathrm{PaO}_{2}(\mathrm{mmHg})$ & $65.64 \pm 4.17$ & $80.65 \pm 6.66$ & $71.1 \pm 3.73$ \\
\hline Mean daytime $\mathrm{PaCO}_{2}(\mathrm{mmHg})$ & $39.78 \pm 2.77$ & $38.37 \pm 3.13$ & $37.42 \pm 5.16$ \\
\hline Mean $\mathrm{FEV}_{1}(\mathrm{ml})$ & $1609 \pm 371.7$ & $1028 \pm 301$ & $1180 \pm 245.7$ \\
\hline Mean $\mathrm{FEV}_{1}$ (\% predicted) & $54.36 \pm 15.37$ & $40.22 \pm 12.5$ & $38.8 \pm 10.6$ \\
\hline Mean BMI $\left(\mathrm{kg} / \mathrm{m}^{2}\right)$ & $27.9 \pm 3.12$ & $21.04 \pm 1.52$ & $21.26 \pm 2.6$ \\
\hline
\end{tabular}

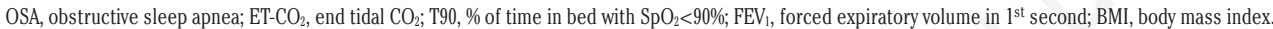

Table 2. Statistical significance (p-values) of the differences in various parameters in the COPD-sleep phenotypes.

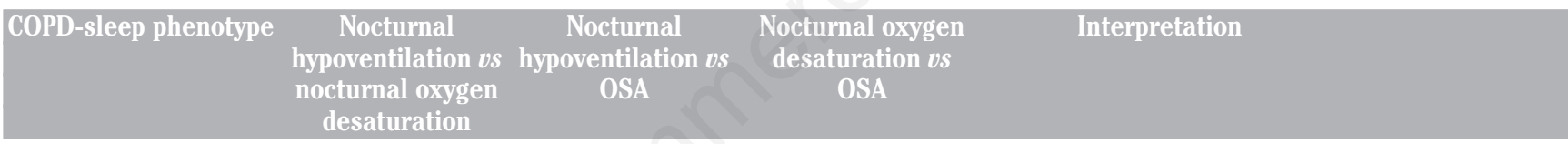

\begin{tabular}{|c|c|c|c|c|}
\hline Mean nocturnal $\mathrm{SpO}_{2}$ & 0.007 & 0.07 & 0.04 & $\begin{array}{l}\text { The mean nocturnal } \mathrm{SpO}_{2} \text { was significantly lower } \\
\text { in nocturnal oxygen desaturation compared to } \\
\text { nocturnal hypoventilation and OSA }\end{array}$ \\
\hline Mean nocturnal ET-CO 2 & 0.001 & 0.001 & 0.09 & $\begin{array}{l}\text { The mean nocturnal ET- } \mathrm{CO}_{2} \text { was significantly } \\
\text { higher in nocturnal hypoventilation compared to } \\
\text { OSA and nocturnal oxygen desaturation }\end{array}$ \\
\hline Mean night-time T90 (\% TIB) & 0.001 & 0.001 & 0.001 & $\begin{array}{l}\text { The mean night time T90 was significantly higher in } \\
\text { nocturnal oxygen desaturation compared to } \\
\text { nocturnal hypoventilation and OSA }\end{array}$ \\
\hline Mean daytime $\mathrm{SpO}_{2}(\%)$ & 0.002 & 0.001 & 0.22 & $\begin{array}{l}\text { The mean daytime } \mathrm{SpO}_{2} \text { was significantly lower in } \\
\text { nocturnal oxygen desaturation and OSA compared } \\
\text { to nocturnal hypoventilation }\end{array}$ \\
\hline Mean daytime $\mathrm{PaO}_{2}(\mathrm{mmHg})$ & 0.01 & 0.001 & 0.2 & $\begin{array}{l}\text { The mean daytime } \mathrm{PaO}_{2} \text { was significantly lower in } \\
\text { nocturnal oxygen desaturation and OSA compared } \\
\text { to nocturnal hypoventilation }\end{array}$ \\
\hline Mean daytime $\mathrm{PaCO}_{2}(\mathrm{mmHg})$ & 0.67 & 0.29 & 0.24 & $\begin{array}{l}\text { There was no significant difference in mean } \\
\text { daytime } \mathrm{PaCO}_{2} \text { between the three phenotypes }\end{array}$ \\
\hline Mean $\mathrm{FEV}_{1}(\mathrm{ml})$ & 0.35 & 0.001 & 0.03 & $\begin{array}{l}\text { The mean } \mathrm{FEV}_{1}(\mathrm{ml}) \text { was significantly higher in } \\
\text { OSA compared to nocturnal hypoventilation and } \\
\text { nocturnal oxygen desaturation }\end{array}$ \\
\hline Mean $\mathrm{FEV}_{1}$ (\% predicted) & 0.83 & 0.001 & 0.04 & $\begin{array}{l}\text { The mean } \mathrm{FEV}_{1} \text { (\% predicted) was significantly } \\
\text { higher in OSA compared to nocturnal } \\
\text { hypoventilation and nocturnal oxygen desaturation }\end{array}$ \\
\hline Mean BMI $\left(\mathrm{kg} / \mathrm{m}^{2}\right)$ & 0.84 & 0.001 & 0.001 & $\begin{array}{l}\text { The mean BMI was significantly higher in OSA } \\
\text { compared to nocturnal hypoventilation and } \\
\text { nocturnal oxygen desaturation }\end{array}$ \\
\hline
\end{tabular}


they found that $22 \% \mathrm{COPD}$ patients having a daytime $\mathrm{PaO}_{2} \geq 60$ $\mathrm{mmHg}$, desaturated below a baseline of $90 \%$ for $\geq 30 \%$ of their sleep time. Some other studies have found that $27-70 \%$ of COPD patients with the daytime saturations between $90 \%$ and $95 \%$ have nocturnal oxygen desaturation [13-16]. But in these studies, the contribution of nocturnal hypoventilation leading to hypoxemia have not been evaluated. We found that though nocturnal hypoxemia was seen in 17 (20\%) patients with daytime $\mathrm{PaO}_{2}>60 \mathrm{~mm}$ of $\mathrm{Hg}$, the majority $70 \%$ $(12 / 17)$ had nocturnal hypoventilation.

We also found that $21(24.7 \%)$ patients with normal day time $\mathrm{CO}_{2}$ had a presence of sleep hypoventilation. There has been no study, which has evaluated nocturnal hypoventilation in presence of normal daytime $\mathrm{CO}_{2}$. Though a study by O'Donoghue et al. [17] has shown that $43 \%$ of stable hypercapnic COPD patients had nocturnal hypoventilation.

Another significant finding in our study was that 17/85 (20\%) patient had OSA but, 10 of them did not have either nocturnal hypoventilation or nocturnal oxygen desaturation. The association between COPD and OSA in the same patient, known as overlap syndrome is common. The prevalence of overlap syndrome varies from $2.9 \%$ to $65.9 \%$ in COPD patients [18]. Our study clearly brings out the different characteristics of patients having nocturnal gas exchange abnormality, which can be termed as "COPD sleep phenotypes". Differentiating OSA, nocturnal hypoventilation and nocturnal oxygen desaturation into 3 different phenotypes is a novel thing. We compared the three phenotypes among themselves in terms of mean BMI, mean $\mathrm{SpO}_{2}$, mean $\mathrm{PaO}_{2}$, mean $\mathrm{PaCO}_{2}$, and mean $\mathrm{FEV}_{1}$. In the patients who had nocturnal oxygen desaturation, their day time $\mathrm{PaO}_{2}$ and $\mathrm{SpO}_{2}$ was significantly lower than those who had nocturnal hypoventilation. The BMI and $\mathrm{FEV}_{1}$, however were similar. This means that the patients with nocturnal hypoventilation and nocturnal oxygen desaturation may have different ability to respond to hypercapnia at night. Those with nocturnal hypoventilation have poor response to elevation of nocturnal $\mathrm{CO}_{2}$ compared to nocturnal oxygen desaturation phenotype. The patients who have nocturnal hypoventilation would be unable to maintain their normal daytime $\mathrm{CO}_{2}$ levels soon. There is evidence regarding the fact that increased $\mathrm{CO}_{2}$ levels cause blunting of the chemoreceptor response [19]. This blunted response to increased nocturnal $\mathrm{CO}_{2}$ subsequently causes day time hypercapnia and

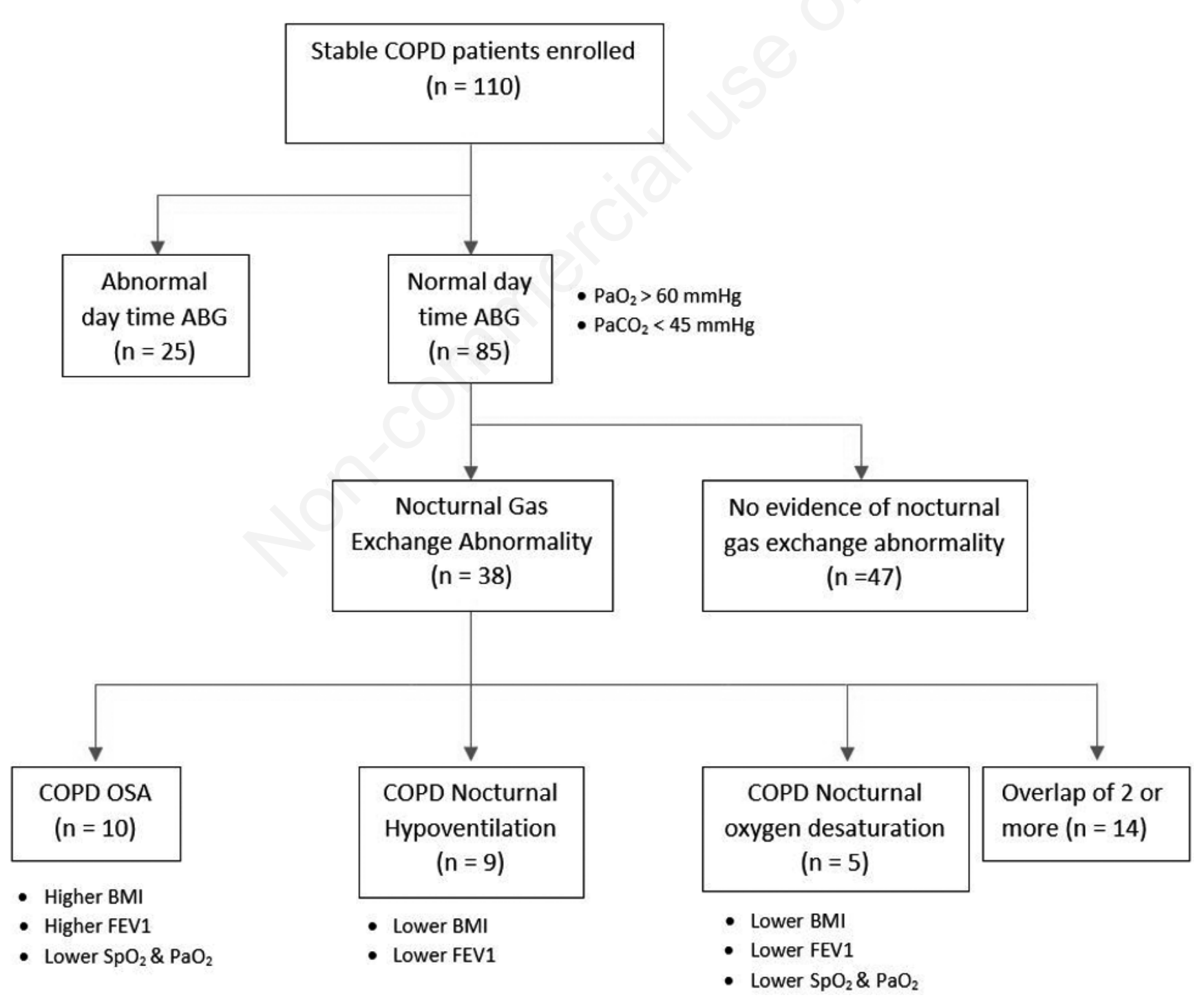

Figure 2. Flow diagram showing classification of COPD phenotypes. 
hence overt respiratory failure. This process of daytime hypercapnia can be delayed if they are treated early with bilevel positive airway pressure (BPAP). We found a striking difference in the spirometry and blood gas profile of patients having OSA compared to those having nocturnal oxygen desaturation or nocturnal hypoventilation. The patients with OSA had a statistically significant higher mean BMI and mean $\mathrm{FEV}_{1}$ as compared to those who had nocturnal hypoventilation or nocturnal oxygen desaturation. This indicates that patients having OSA have a preserved lung functions and higher BMI. In a study by Schreiber et al. [20] patients having OSA were found to have less severe airway obstruction which is also seen in our study. The presence of low BMI is protective for OSA [21]. Since 10/17 OSA patients did not have hypoxia or hypoventilation they can be treated with continuous positive airway pressure therapy (CPAP). Those with desaturation persisting on CPAP may require additional oxygen. Those with overlap of hypoventilation will require BPAP if their nocturnal $\mathrm{CO}_{2}$ did not improve with CPAP alone.

To conclude the COPD patients going into respiratory failure pose a huge burden on health care system. The various clinical characteristics can help in early detection of the different phenotypes of COPD patients and subject them to appropriate nocturnal investigation $\left(\mathrm{O}_{2}\right.$ and/or $\mathrm{CO}_{2}$ monitoring or PSG). These investigations can define the treatment options for different diseases in terms of CPAP, BPAP or nocturnal oxygen therapy [9]. Thus, it is important to perform the sleep evaluation at the time of diagnosis of COPD and then annually. A treatment as per the respective phenotypes is likely to prevent the progression of disease and development of respiratory failure. Further research with case-control study and follow up is needed to see the response to treatment.

\section{Limitation of the study}

Our study is the first of its kind and brings out a new perspective, but it has a few shortcomings. The logistics issues prevented us from performing level 1 polysomnography on all the patients. It is an observational study with a small sample size. The patients need to be followed up for seeing the progression of disease.

\section{References}

1. McNicholas WT, Verbraecken J, Marin JM. Sleep disorders in COPD: the forgotten dimension. Eur Respir Rev 2013;22:365-75.

2. Gibson GJ. Clinical Tests of Respiratory Function. 3rd ed. London: Hodder Arnold; 2009.

3. Kent BD, Mitchell PD, McNicholas WT. Hypoxemia in patients with COPD: cause, effects, and disease progression. Int J Chron Obstruct Pulmon Dis 2011;6:199-208.

4. Sarkar M, Niranjan N, Banyal PK. Mechanisms of hypoxemia. Lung India 2017;34:47-60

5. Flenley DC. Sleep in chronic obstructive lung disease. Clin Chest Med 1985;6:651-61.
6. Miller MR, Hankinson J, Brusasco V, et al. ATS/ERS task force: standardisation of lung function testing. Eur Respir $\mathrm{J}$ 2005;26:319-38.

7. Global Initiative for Chronic Obstructive Lung Disease (GOLD). Global strategy for diagnosis, management, and prevention of COPD, 2018. Accessed on May 31, 2019. Available from: http://goldcopd.org

8. No authors listed. Sleep-related breathing disorders in adults: recommendations for syndrome definition and measurement techniques in clinical research: the report of an American Academy of Sleep Medicine Task Force. Sleep 1999;22:667-89.

9. Budhiraja R, Siddiqi TA, Quan SF. Sleep disorders in chronic obstructive pulmonary disease: etiology, impact, and management. J Clin Sleep Med 2015;11:259-70.

10. Berry RB, Budhiraja R, Gottlieb DJ, et al. Rules for scoring respiratory events in sleep: update of the 2007 AASM Manual for the Scoring of Sleep and Associated Events. Deliberations of the Sleep Apnea Definitions Task Force of the American Academy of Sleep Medicine. J Clin Sleep Med 2012;8:597-619.

11. Fletcher EC, Scott D, Qian W, et al. Evolution of nocturnal oxyhemoglobin desaturation in patients with chronic obstructive pulmonary disease and a daytime $\mathrm{PaO} 2$ above 60 torr. Am Rev Respir Dis 1991;144:401-25.

12. Fletcher EC, Donner CF, Midgren B et al. Survival in COPD patients with a daytime $\mathrm{PaO} 2>60 \mathrm{mmHg}$ with and without nocturnal oxyhaemoglobin desaturation. Chest 1992;101:649-55.

13. Angelis GD, Sposato B, Mazzei L, et al. Ondexes of nocturnal desaturation in COPD patients not treated with long term oxygen therapy. Eur Rev Med Pharmacol Sci 2001;5:173-9.

14. Chaouat A, Weitzenblum E, Kessler R, et al. Sleep related O2 desaturation and daytime pulmonary haemodynamics in COPD patients with mild hypoxaemia. Eur Respir J 1997;10:1730-5.

15. Fletcher EC, Schaaf JW, Miller J, et al. Long-term cardio-pulmonary sequelae in patients with sleep apnea and chronic lung disease. Am Rev Respir Dis 1987;135:525-33.

16. Lewis CA, Fergusson W, Eaton T, et al. Isolated nocturnal desaturation in COPD: prevalence and impact on quality of life and sleep. Thorax 2009;64:133-8.

17. O'Donoghue FJ, Catcheside PG, Ellis EE, et al. Sleep hypoventilation in hypercapnic chronic obstructive pulmonary disease: prevalence and associated factors. Eur Respir J 2003;21:977-84.

18. Shawon MS, Perret JL, Senaratna CV, et al. Current evidence on prevalence and clinical outcomes of co-morbid obstructive sleep apnea and chronic obstructive pulmonary disease: a systematic review. Sleep Med Rev 2017, 32:58-68.

19. Scano G, Spinelli A, Duranti R, et al. Carbon dioxide responsiveness in COPD patients with and without chronic hypercapnia. Eur Respir J 1995;8:78-85.

20. Schreiber A, Cemmi F, Ambrosino N, et al. Prevalence and predictors of obstructive sleep apnea in patients with chronic obstructive pulmonary disease undergoing inpatient pulmonary rehabilitation. COPD 2018;15:265-70.

21. McNicholas WT. Comorbid obstructive sleep apnoea and chronic obstructive pulmonary disease and the risk of cardiovascular disease. J Thorac Dis 2018;10:S4253-61. 